\title{
The reproducibility of optical mammography in healthy volunteers
}

\author{
L C Enfield, J C Hebden and A P Gibson ${ }^{1}$ \\ Department of Medical Physics and Bioengineering, UCL, Gower Street, London WC1E 6BT, \\ UK \\ E-mail: adam.gibson@ucl.ac.uk
}

Received 20 December 2012, in final form 14 May 2013

Published DD MMM 2013

Online at stacks.iop.org/PMB/58/1

\begin{abstract}
This study was designed to determine the reproducibility of optical mammography. Eight healthy pre-menopausal volunteers were scanned at different time intervals (minutes, weeks and months apart) to investigate the effects of within-subject variation, between-subject variation and systematic variations on both the raw data and images. The study shows that the greatest source of variation in optical mammography raw data and images is between different subjects, and scans of the same subject are very reproducible. The averaged total haemoglobin concentration from the eight volunteers was $(24 \pm 10) \mu \mathrm{M}$, and the average tissue oxygen saturation was $(70 \pm 10) \%$, which is comparable with other data in the literature. The average absorption coefficient at $780 \mathrm{~nm}$ was $(0.0048 \pm 0.0017) \mathrm{mm}^{-1}$ and the average reduced scatter coefficient at $780 \mathrm{~nm}$ was $(0.80 \pm 0.12) \mathrm{mm}^{-1}$. Again, this is comparable with published values. When our data are combined with the published values, the weighted average total haemoglobin concentration and tissue oxygen saturation for pre-menopausal breasts are $(29 \pm 8) \mu \mathrm{M}$ and $(73 \pm 3) \%$ respectively. The results of our study show that we can be reassured that any changes within the tumour region seen during neoadjuvant therapy are likely to be due to a real physiological response to treatment, as the physiological properties of the breast remain relatively constant. However, in this study, we cannot distinguish between a tumour response to treatment and systemic changes in the healthy breast.
\end{abstract}

(Some figures may appear in colour only in the online journal)

1 Author to whom any correspondence should be addressed. 


\section{Introduction}

Near-Infrared (NIR) optical tomography has increasingly been used to investigate the response of patients to primary medical therapy for locally advanced breast cancer, with papers appearing in the literature since 2004 (Jakubowski et al 2004). Several reviews of the topic have been published, each giving an overview of the current research (Enfield et al 2009, Choe and Durduran 2012). Assessing the response to treatment could be an ideal application for optical methods, as there is less need for high spatial resolution. The ability to perform multiple scans over a period of time and acquire physiological information cheaply and safely plays to the strengths of the technique.

The reproducibility of images is obviously of great importance when conducting longitudinal studies, to ensure that any changes seen in the optical parameters are genuinely due to physiological factors. There has been no published investigation into the reproducibility of optical images of the breast. This study aims to investigate possible sources of variation that may occur when carrying out optical mammography. We used the UCL MONSTIR imaging system (Schmidt et al 2000) and a liquid coupled volunteer interface (Yates et al 2005b) to collect optical mammography data from healthy pre-menopausal volunteers.

Variation can come from three different sources: within-subject variations, betweensubject variations and systematic variations from the hardware, imaging equipment, data processing or image reconstruction. Some of these sources of variation may be specific to a particular system, but others are general.

Using our system, within-subject variations may be due to the positioning of the volunteer in the patient interface, or from physiological changes within the breast due to the menstrual cycle. Some studies have reported changes in physiological properties over the course of the menstrual cycle with Shah et al (2001) reporting an increase in total haemoglobin concentration [HbT] of $(10.63 \pm 0.05) \mu \mathrm{M}$ (mean and standard deviation (S.D.)) from on day 14, to (15.76 \pm 0.08$) \mu \mathrm{M}$ on day 28 in a single subject. Pogue et al (2004) found a $13 \%$ increase in haemoglobin in the second half of the cycle when compared to the first half of the cycle in seven women. However, other studies have proved inconclusive when looking at changes in [HbT] over the menstrual cycle (Cubeddu et al 2000, Yates 2005a).

Sources of between-subject variation include breast density and menopausal status. Denser breasts contain greater proportions of water, lipid and [HbT] and exhibit greater scattering (Spinelli et al 2004, Srinivasan et al 2003). Published literature suggests that premenopausal women have higher concentrations of haemoglobin compared to post-menopausal women $((34 \pm 9) \mu \mathrm{M}$ versus $(14 \pm 0) \mu \mathrm{M})$, and have more heterogeneous breasts (Leff et al 2008).

Systematic variations can either be generic, or specific to a particular imaging system. Generic sources of variation include laser power fluctuations, coupling light to and from the patient/volunteer, variation in the optical properties of coupling fluid (if used), and electronic jitter introducing uncertainties into measurements of mean photon flight time. Specific sources of variation with our system are mainly due to the variable optical attenuators (VOAs).

The VOAs control the amount of light reaching the microchannel-plate photomultiplier tubes (MCP-PMTs) and protect them from over-illumination. They are small rotating discs with a range of different size holes in them (Schmidt et al 2000). The VOAs are moved from position to position by stepper motors. There are sometimes variations in the alignment between the apertures in the VOA and the detector fibres at the smaller apertures sizes due to the stepper motor step-size. This can lead to either an increased or decreased amount of light reaching the detector, distorting the intensity measurements. Testing of the VOAs with 
repeated measurements over one hour have also shown variations in the attenuation provided, resulting in a roughly $4 \%$ error in intensity measurements (Jennions 2008).

\section{Methods}

\subsection{Imaging system}

The UCL 32-channel time resolved optical imaging system (MONSTIR) uses a portable fibre laser (IMRA Inc., USA) which produces pulses $\sim 2 \mathrm{ps}$ in duration at 780 and $815 \mathrm{~nm}$, interlaced at a repetition rate of $80 \mathrm{MHz}$ (Yates et al 2005b). Photons transmitted through the breast and coupling liquid are collected by optical fibre bundles coupled to MCP-PMTs that produce an electronic pulse for each detected photon. A histogram of photon arrival times, known as a temporal point spread function (TPSF) is generated for each source-detector combination by timing electronics (Becker \& Hickl GmbH, Berlin, Germany). Calibration data were also collected and these were used to deconvolve the effects of fibre length and internal reflections from each measured TPSF (Hebden et al 2003). Images are then reconstructed using data types extracted from the TPSFs. The mean photon flight time and intensity data types are selected for reconstruction, as they are the most robust and reliable particularly when the number of photons recorded is low (Yates et al 2005c).

\subsection{Volunteer interface}

The volunteer interface consists of a $160 \mathrm{~mm}$ diameter rigid hemisphere with 31 regularly spaced $1 \mathrm{~cm}$ diameter holes with Perspex windows fixed into each hole. The optical fibre bundles are attached to the exterior surface of the cup by inserting them within short plastic tubes fixed over each window. The cup is attached to a plastic ring which fits within an aperture in a wooden table, as shown in figure 1. A coupling fluid fills the remaining space between the breast and the hemisphere. The volunteer lies prone with her breast pendant in the liquid coupled interface. For more details on the interface and the coupling fluid, please see Yates et al (2005b) and Enfield et al (2007).

The coupling fluid was designed to have optical properties of absorption coefficient $\left(\mu_{\mathrm{a}}\right)=0.004 \mathrm{~mm}^{-1}$ and reduced scatter coefficient $\left(\mu_{\mathrm{s}}^{\prime}\right)=0.8 \mathrm{~mm}^{-1}$ at $780 \mathrm{~nm}$. The coupling fluid consists of Intralipid (a soya based fat emulsion) with NIR-absorbing dye and water. It was mixed in the same proportions for each scan. In each case, four litres of the coupling fluid were made up, consisting of ICI S109564 absorbing dye (ICI, London, UK), 20\% Intralipid (Fresenius $\mathrm{KABI} U K$, Runcorn, $\mathrm{UK}$ ) and deionized water. It was maintained at a constant temperature of $37^{\circ} \mathrm{C}$ using an electrically-isolated water heater.

As reported previously, we reconstruct the changes in optical properties between the cup containing both the coupling fluid and the breast and those of the cup containing the fluid alone as a reference (Yates et al 2005b). This eliminates the effect of uncertainty and variability in the surface coupling and allows the use of relative intensity measurements as well as difference in mean photon flight time in the reconstruction. However, it does create a refractive mismatch index between the breast (generally assumed to be about 1.4) and the liquid ( $n=1.36$ (Ding et al 2005)). Simulations studies suggest that under normal circumstances, the effect of this mismatch on the reconstructed images is small and can be neglected (Dehghani et al 2005). Detector gains, the source/detector fibre lengths and positions, and the laser source power were kept constant for all scans. 


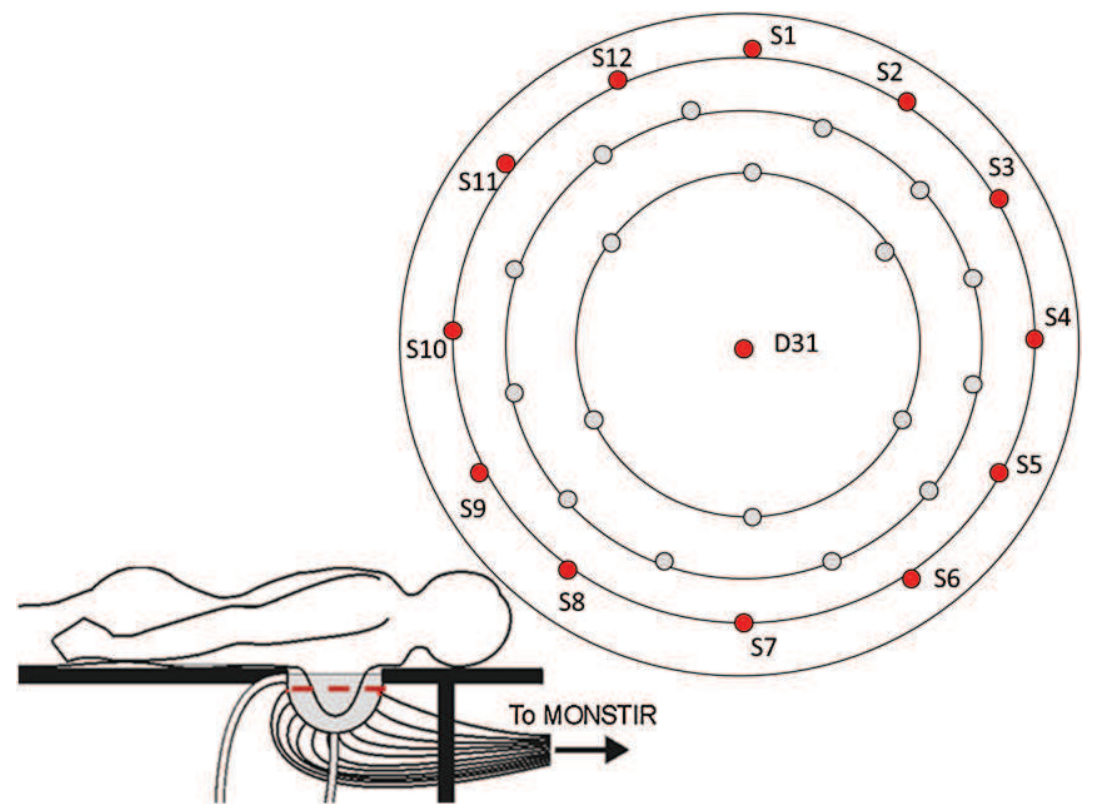

Figure 1. The fluid-coupled volunteer interface for 3D optical breast imaging. The volunteer lies with her breast pendant in a hemispherical cup filled with coupling fluid. 30 optode connectors are arranged in three concentric rings with one further optode at the bottom of the imaging cup. Each optode acts as both a source and detector. S1 to S12 are in the top ring of optode connectors, while $\mathrm{D} 31$ is at the bottom of the imaging cup.

\subsection{Image reconstruction}

Three-dimensional (3D) images representing variation in $\mu_{\mathrm{a}}$ and $\mu^{\prime}$ s were generated using the TOAST reconstruction package (Arridge and Schweiger 1997). The finite element method was used to simulate photon propagation in the hemispherical tank using a finite element mesh with 67537 nodes and 46415 tetrahedral elements. The nonlinear inverse problem was solved by iteratively adjusting the internal properties using conjugate gradients to minimize an error norm between the model and the data. In practice, the reconstruction was stopped either after 15 iterations or before 15 iterations if the error norm no longer reduced significantly. The starting values for the iterative model were taken to be the measured absorption and scatter coefficients of the coupling fluid.

We assume that the optical absorption represented in the absorption images is due to three components: oxyhaemoglobin $\left(\mathrm{HbO}_{2}\right)$, deoxyhaemoglobin $(\mathrm{Hb})$, and a background component with the same absorption coefficient at both wavelengths (assumed to be predominately due to lipid and water, but with contributions from other chromophores e.g. proteins, DNA, melanin and cytochrome oxidase) (Matcher et al 1994). The background component was assumed to contribute $70 \%$ of the total absorption, based on previous findings (Enfield et al 2007). Changes in total haemoglobin concentration $[\mathrm{HbT}]$ and tissue oxygenation saturation $\left(\mathrm{SatO}_{2}\right)$ can be derived from the absorption images and prior knowledge of the extinction coefficients of $\mathrm{HbO}_{2}$ and $\mathrm{Hb}$. We assume the initial total haemoglobin concentration is $14 \mathrm{~g} \mathrm{dl}^{-1}(2 \mathrm{mM})$, based on the normal range in blood in the healthy adult female of $12.2-15.0 \mathrm{~g} \mathrm{dl}^{-1}(1.9-$ $2.3 \mathrm{mM}$ ) (Hillman 2002). 
Table 1. Protocol of scans used to investigate sources of variation in optical mammography. Eight volunteers took part in the project.

\begin{tabular}{llll}
\hline Volunteer & Age & Scanning protocols & $\begin{array}{l}\text { Number of useable } \\
\text { image sets obtained }\end{array}$ \\
\hline 1 & 28 & Monthly for three months & 6 \\
2 & 28 & Multiple scans in one imaging session & 8 \\
3 & 36 & Monthly for three months & 6 \\
4 & 23 & Weekly for four weeks & 8 \\
5 & 33 & Monthly for three months & 6 \\
6 & 31 & Multiple scans in one imaging session & 8 \\
& & Weekly for four weeks & 8 \\
7 & 23 & Multiple scans in one imaging session & 8 \\
8 & 25 & Weekly for four weeks & 8 \\
\hline
\end{tabular}

\subsection{Imaging protocols}

Eight healthy volunteers aged 23-36 (29 \pm 5 (mean \pm S.D.)) years old were scanned on three different images protocols (table 1). One volunteer was scanned on two different protocols. The three protocols were as follows:

(1) Four times in one imaging session, to allow us to look at between-subject variations, and short-term systematic variation (such as the laser power). The subject sat up between each scan before lying back down and repositioning herself. The same batch of coupling fluid was used for each of the eight scans $(n=3)$.

(2) Weekly for four weeks, to allow us to look at between-subject variations, and withinsubject variation in a single subject due to the menstrual cycle $(n=3)$. A new batch of coupling fluid, though made to the same recipe, was used for each scan.

(3) Monthly for three months, to allow us to look at long term systematic variation, and between-subject variations $(n=3)$. A new batch of coupling fluid was used for each scan.

A total of 28 imaging sessions were conducted, producing a total of 68 image sets. The day of the volunteer's menstrual cycle was noted when volunteers were scanned once a week for a month. The body mass index (BMI) of each volunteer was calculated, and the mean and S.D. was $(22.8 \pm 2.5)$. Both breasts were scanned in each scanning session. The volume of Intralipid displaced by each volunteer was also recorded to give a measure of breast volume. Each scan took 3 min.

The following six sections describe separate investigations of variability, including methods and results, as follows:

(1) Variations in the coupling fluid used in the volunteer interface

(2) Equipment variation in the raw data

(3) Variation within subjects in the raw data

(4) Variation between subjects in the raw data

(5) Variations in the physiological properties from the images within subjects

(6) Variations in the physiological properties from the images between subjects

Presenting the results in this order enables us to use the results of one investigation to interpret the results of the next. For example, if there are statistically significant variations between batches of coupling fluid, this will affect the results found when looking at between-subject variation. Both the raw data and the images were analysed to study the effects of data processing 
on the reproducibility of the images. Statistics were calculated using IBM SPSS (IBM, Armonk, New York US) and results were deemed to be significant if $p<0.05$. Although data were acquired at $780 \mathrm{~nm}$ and at $815 \mathrm{~nm}$, in this paper we analyse data taken at $780 \mathrm{~nm}$ only, for clarity.

\section{Variations in optical properties of the coupling fluid used in the volunteer interface}

\subsection{Introduction}

While the optical properties of the coupling fluid used in the liquid-filled patient interface should remain the same every time a new solution is generated, variation may arise between different batches of Intralipid, or due to errors in the measurement of volume. For these reasons, industrial groups such as Philips have discontinued the use of Intralipid as a coupling medium. It is important that the coupling fluid properties are reproducible, as variations in the absorption and scatter between scans will affect any repeated measurements made on patients or volunteers.

\subsection{Methods}

Data were collected on the coupling fluid-filled cup alone once a month for nine months. The $\mu_{\mathrm{a}}$ and $\mu_{\mathrm{s}}$ of the coupling fluid were measured after each scan. The properties were measured using a single source and a single detector on opposite sides of a $54 \mathrm{~mm}$ diameter Perspex box, and collecting a TPSF, averaging for $30 \mathrm{~s}$. A Green's function solution to the time-dependent diffusion equation was fitted to the measured TPSF using the least squares quadratic method to find the $\mu_{\mathrm{a}}$ and $\mu^{\prime}{ }_{\mathrm{S}}$ of the coupling fluid (Hebden and Delpy 1994). The Intralipid used was extracted from different sealed packs, but were all from the same manufacturing batch. A one sample $t$-test was performed to compare the coupling fluid optical properties to the intended value. For $\mu_{\mathrm{a}}$ this was $0.004 \mathrm{~mm}^{-1}$ and for $\mu^{\prime}$ s it was $0.8 \mathrm{~mm}^{-1}$.

\subsection{Results}

The measured $\mu_{\mathrm{a}}$ for the nine samples was $(0.0044 \pm 0.0001) \mathrm{mm}^{-1}$ (mean \pm S.D.), and the measured $\mu^{\prime}$ s was $(0.83 \pm 0.02) \mathrm{mm}^{-1}$ at $780 \mathrm{~nm}$. There was no significant difference in either the absorbing or the scattering properties of the coupling fluid over the nine scans $(p=0.08$ and $p=0.175)$.

\subsection{Discussion}

While mixing the coupling fluid, small variations in the relative concentrations will occur. However, these variations do not have a statistically significant effect on the optical properties. This is important, as it means that we can exclude variations in the coupling fluid as a possible source of within-subject and between-subject variations.

\section{Systematic variation}

\subsection{Introduction}

Systematic changes in the equipment were examined by collecting and analysing data from the coupling fluid-filled volunteer interface alone (with no volunteer breast in place). The 
previous section showed that the coupling fluid properties are reproducible between scans, so any remaining variation is likely to be due to changes in the instrumentation.

\subsection{Methods}

Data were collected from the coupling fluid-filled cup alone once a month for nine months. The absolute mean photon flight time and intensity data from 12 different optical sources (sources 1 to 12) and one detector (detector 31) from each scan were averaged together. These twelve sources are arranged in a single ring near the top of the imaging cup, and are all equidistant from the detector $(46 \pm 3 \mathrm{~mm}$ ) (shown in red in figure 1). The detector is positioned at the bottom of the cup (D31 in figure 1).

Mean time and intensity data types are extracted from the deconvolved TPSFs collected on the volunteer, and on the coupling fluid-filled cup alone. Each TPSF was assessed and rejected if it was distorted, for example by pre-peaks or post-peaks which can be due to multiple reflections, or if less than one hundred counts were detected. Then the extracted data types were examined by plotting the mean tine or log intensity against optode separation. Any points which deviated substantially away from an approximately linear trend were also rejected. This may occur due to errors introduced by the deconvolution, low photon counts or high levels of noise on a particular source-detector combination. For example, we acquire 900 source-detector pairs. In typical analysis of a standard data set, 126 measurements were excluded following examination of the TPSFs and a further 289 points were rejected following examination of the data types, leaving 485 measurements for subsequent analysis and reconstruction.

The reproducibility of the absolute mean photon flight time data and intensity data was analysed using ANOVA. The null hypothesis was that the means of the intensity data and mean photon flight time data from each of the six data sets are equal.

\subsection{Results}

The variations in the absolute mean photon flight time and intensity data from the monthly scans on the coupling fluid-filled cup were calculated and are displayed in figure 2 . The results from each scan and the mean and S.D. for all the scans combined is given.

Data from scans taken at months 1,2 and 9 were excluded as more than half of the data points (from sources 1-12 with detector 31) per scan were rejected using our exclusion criteria. The remaining six sets of scan data revealed no significant difference between the mean photon flight time data $(p=0.08)$, but there were significant differences in the intensity data $(p=0.02)$. The mean and S.D. for mean photon flight times over the six scans is $(2675 \pm 63)$ ps. The mean and S.D. for intensity over the six scans is $(2226 \pm 471)$ photons.

\subsection{Discussion}

We believe the main source of systematic variation is due to the VOAs in the MONSTIR imaging system, and the effect that VOA misalignment at the smaller aperture sizes has on the intensity measurements (Jennions 2008). Since these data were acquired, we have developed a new version of our imaging system. It also has VOAs, but they have been designed to combat the problem identified. The new VOAs are made of discs from x-ray film, with ten different variably-exposed sections. Each section's NIR attenuation depends on the density of the developed film (Jennions 2008). The sections are much larger than the small holes in the old design, which will reduce the variation in the intensity data caused by the variation in the alignment of the smaller apertures in the VOAs. Testing of the new VOAs with repeated measurements have shown a $<1 \%$ error in intensity measurements (Jennions 2008). 

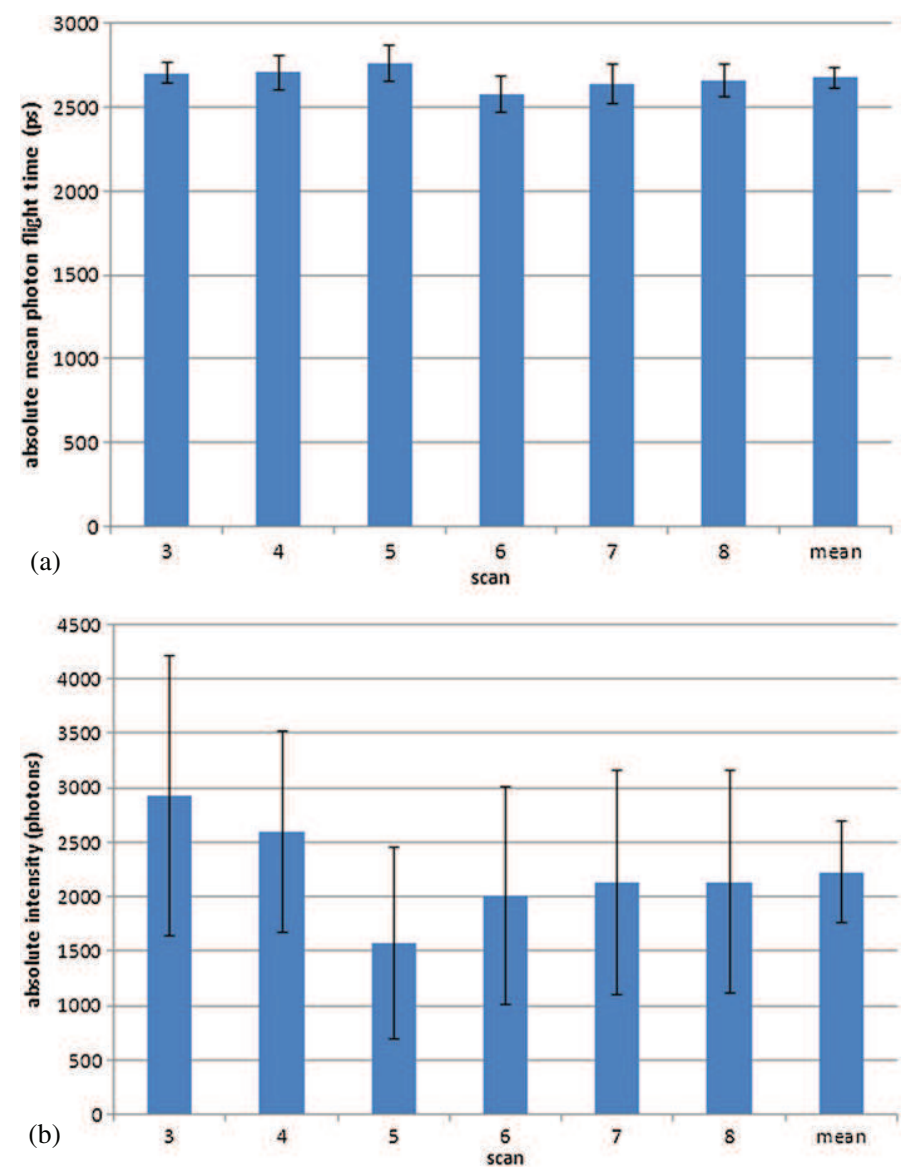

Figure 2. (a) The absolute mean photon flight time data and (b) the absolute intensity data collected on the coupling fluid-filled imaging cup over six scans. The error bars show one S.D.

\subsection{Summary of systematic variation}

In summary, there is no variation in the absorption and scattering properties of the coupling fluid used in the volunteer interface. There is a statistical significant variation in the intensity measurements made on the coupling fluid-filled interface over the course of scans performed monthly, but not in the mean photon flight time data.

\section{Within-subject variation}

\subsection{Introduction}

We have established that the optical properties of the Intralipid coupling fluid are constant, and characterised the variation in the intensity measurements due to the imaging system itself. This means that we can now better characterise any differences in data sets collected on the same volunteer. Assessing the raw data gives us a direct measure of the data reproducibility without any additional uncertainty caused by the image reconstruction.

There is heterogeneity within in the optical properties of the breast in an individual premenopausal woman (Yates et al 2005c). There will also be variation due to the positioning of 
the subject in the liquid-coupled interface. While the diameter of the imaging cup (160 mm) does not allow much movement, the subject will be in a slightly different position for each scan.

\subsection{Methods}

The difference data used for image reconstruction are the mean photon flight times collected on the coupling fluid-filled cup alone subtracted from the mean photon flight times collected on the coupling fluid-filled cup with the volunteer's breast in place and the equivalent difference data for the intensity ratio. The data were processed as described in section 4.2 and analysed with ANOVA.

\subsection{Results}

We compared the data from the left breast against the data from the right breast in all eight volunteers There was no significant difference for either the absolute mean photon flight time $(p=0.492)$ or intensity $(p=0.403)$. There were also no significant differences in the difference mean photon flight time $(p=0.057)$ or difference intensity $(p=0.782)$.

We then looked at the difference between scans on the same volunteers with different time intervals. There were no significant differences in the mean photon flight time and intensity values when multiple scans are conducted in one day. There was a significant difference in the intensity data for both weekly and monthly scans $(p=0.022$ and $p \leqslant 0.0001)$ and in the mean photon flight time for the monthly scans $(p \leqslant 0.0001)$.

\subsection{Discussion}

The variations seen between the weekly and monthly scans could be due to a number of different factors. The volunteer will be in a slightly different position in the imaging cup for each of the scans, which will introduce variation into the data, assuming the optical properties of the coupling medium do not exactly match those of the breast. The range of variation in the position of the breast between scans is limited by the size of the cup (160 mm diameter), but this still allows for some differences in positioning.

It was shown in section 4 that intensity is affected by systematic variation and this can introduce significant variation into the data, especially on the scans conducted months apart. The S.D. for the mean of the intensity measurements made on the coupling fluid-filled up alone (from six monthly scans) was 471 photons per TPSF ( $21 \%$ of the total number of photons when expressed as a percentage). We hypothesised that the intensity differences from the volunteers would be greater, as they would include the variation due to the equipment, and due to the volunteer. It was found that the S.D. for the mean of all the data from all the volunteers was 1107 photons per TPSF (53\% of the total number of photons when expressed as a percentage), suggesting that variation between subjects is a larger source of error than systematic variation.

Durduran et al (2002) looked at variation in measurements made at 17 points on the breast of a volunteer ten times every $2 \mathrm{~min}$. They found that the amplitude data varied by $5-10 \%$, compared to $1-2 \%$ on an Intralipid phantom. This was attributed to physiological variations, such as movement, respiration and heart rate. The UCL system averages data over a $10 \mathrm{~s}$ acquisition, so it can be assumed that the effects of the cardiac and respiratory cycles will average out. Furthermore, the coupling fluid is maintained at $37{ }^{\circ} \mathrm{C}$ to minimise blood flow changes in the skin, suggesting that differences in positioning are a greater source of variation than physiological changes. 
Table 2. The statistical differences for the intra-subject variation in mean difference photon flight time data and intensity data for the processed results collected on healthy volunteers scanned on three different protocols. ${ }^{*}$ denotes $p \leqslant 0.05$.

\begin{tabular}{lll}
\hline & \multicolumn{2}{c}{$\begin{array}{l}p \text { value for differences } \\
\text { between scans }\end{array}$} \\
\cline { 2 - 3 } Imaging protocol & Mean time & Intensity \\
\hline Eight scans in one session & 0.977 & 0.274 \\
Weekly & 0.071 & $0.022^{*}$ \\
Monthly & $\leqslant 0.0001^{*}$ & $\leqslant 0.0001^{*}$ \\
\hline
\end{tabular}

Table 3. The statistical differences for the between-subject variation in the difference mean photon flight time data and intensity data for the processed results collected on healthy volunteers scanned on three difference protocols. ${ }^{*}$ denotes $p \leqslant 0.05$.

\begin{tabular}{lll}
\hline & $\begin{array}{c}p \text { value for differences } \\
\text { between volunteers }\end{array}$ \\
\cline { 2 - 3 } Imaging protocol & Mean time & Intensity \\
\hline Eight scans in one session & $\leqslant 0.001^{*}$ & $\leqslant 0.001^{*}$ \\
Weekly & $\leqslant 0.001^{*}$ & $\leqslant 0.001^{*}$ \\
Monthly & $\leqslant 0.001^{*}$ & $\leqslant 0.001^{*}$ \\
\hline
\end{tabular}

\section{Between-subject variation}

\subsection{Introduction}

It is known that between-subject variations can be significant in both healthy volunteers and in breast cancer patients (Pogue et al 2001, Durduran et al 2002, Srinivasan et al 2003). For this reason, relative measurements such as the contrast between the tumour and the healthy breast tissue or the normalised changes over time are often used when looking at changes within the tumour in response to treatment (Jiang et al 2009). Both the breast radiological density and the BMI have significant effect on the optical properties of the breast, with decreased [HbT] seen in women with a higher BMI compared to those with a lower BMI (Leff et al 2008). Our volunteers had BMIs of between 20 and 26 (22.3 \pm 2.5 mean \pm S.D).

\subsection{Methods}

The raw data were processed and analysed as in section 5.2.

\subsection{Results}

Inter-subject variation is significant for all three imaging protocols for both the mean photon fight time and the intensity data (table 3 ).

\subsection{Discussion}

There was a significant difference between subjects in all of the six data sets examined. This is expected, as we know that there will be significant differences between individuals due to physiological factors such as BMI, breast size and breast density (Leff et al 2008). The 


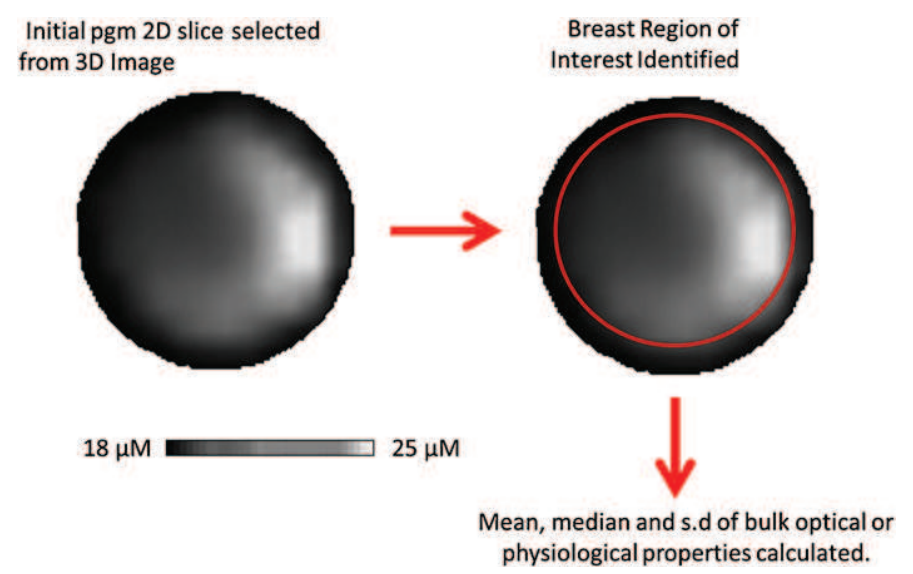

Figure 3. A typical 2D absorption image with the selected breast ROI highlighted in red.

variation in both the intensity and mean photon flight time data is greater than that seen in the data types collected from the interface alone, and when comparing within-subject variations. While our subjects were of comparable BMI, there were variations in bra cup size that will also introduce variation.

\subsection{Summary of results from raw data}

There were no significant differences within single subjects, apart from in the scans conducted at monthly intervals. There were significant differences between subjects regardless of the interval between scans.

\section{Within-subject variation of optical images of the breast}

\subsection{Introduction}

Images were reconstructed from the raw data, and the reproducibility of the images and the quantitative physiological properties extracted from the images was assessed.

\subsection{Methods}

The data were reconstructed into 3D images using TOAST (see section 2.3). A two-dimensional (2D) image was extracted from each 3D reconstructed image of absorption, reduced scatter, [HbT] and $\mathrm{SatO}_{2}$. The 2D coronal slice was taken half way between the chest wall and the nipple. A circular region of interest (ROI) corresponding to the breast was selected, excluding the coupling fluid boundary. Figure 3 shows how the breast ROI was selected from a typical $2 \mathrm{D}$ slice from the 3D image. This boundary was identified in the reconstructed images by the researcher. The same researcher assessed all the images from the volunteers. The mean and S.D. of the bulk optical and physiological values in this breast ROI were calculated. The typical number of pixels in a image slice used to calculate the mean and S.D. was 7000. A two-way ANOVA was used to calculate any statistically significant differences in both [HbT] and $\mathrm{SatO}_{2}$ bulk properties due to the scan number and the subject. 


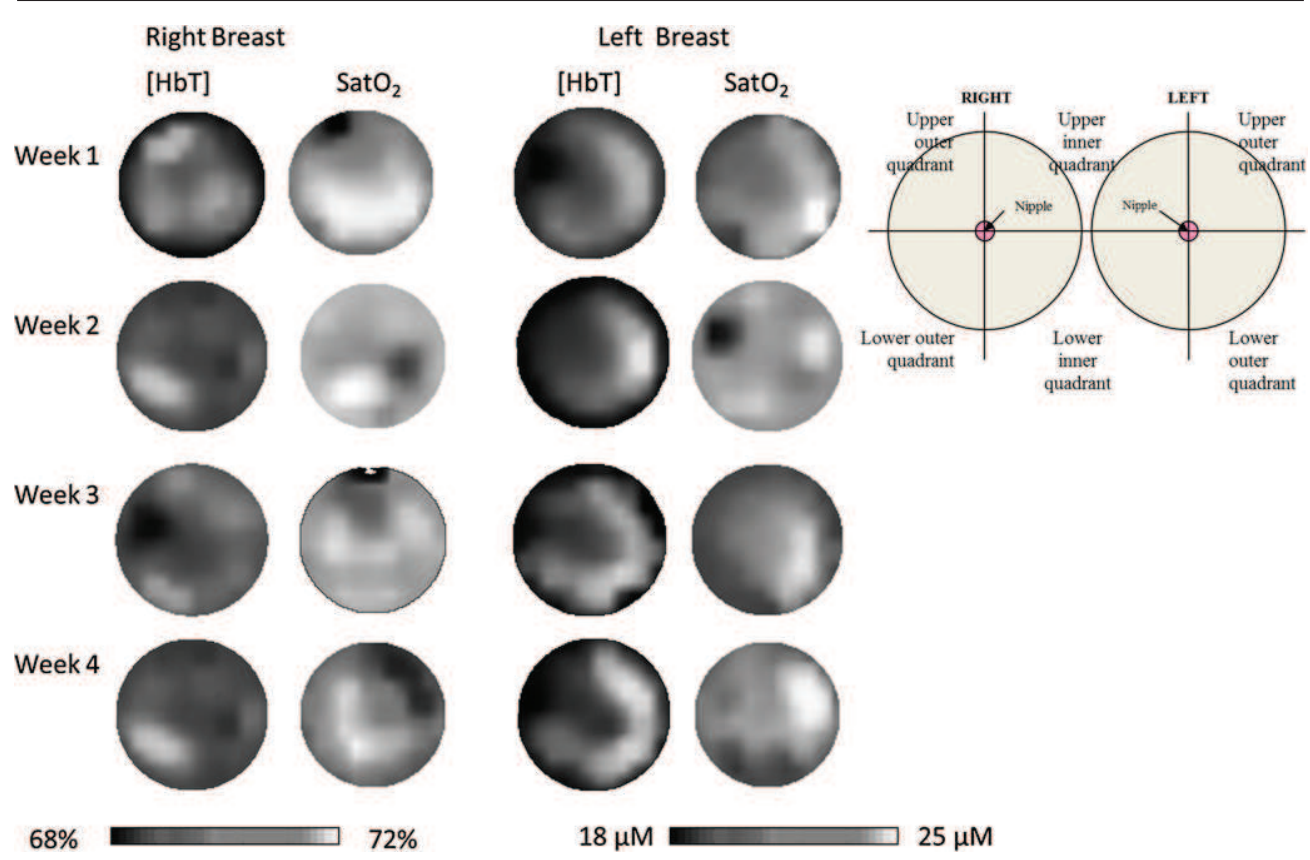

Figure 4. Shows an example of an image set from a volunteer scanned once a week for a month. The images are presented as if the viewer is looking at the subject. The images are adjusted to all be shown on the same scale. The same features are seen in all the [HbT] images, with increased $[\mathrm{HbT}]$ seen in the outer quadrants of both the left and right breast. The $\mathrm{SatO}_{2}$ images are more heterogeneous than the [HbT] images. This heterogeneity is often seen in younger women, due to the physiological makeupof the breast in pre-menopausal women.

\subsection{Results}

3D images were reconstructed from the processed mean photon flight time and intensity difference data, and physiological values were extracted from a $2 \mathrm{D}$ slice of the images The average optical and physiological properties averaged over all women were as follows: absorption coefficient $\left.\left(\mu_{\mathrm{a}}\right)=0.0048 \pm 0.0017\right) \mathrm{mm}^{-1}$; reduced scatter coefficient $\left(\mu_{\mathrm{s}}^{\prime}\right)=$ $(0.80 \pm 0.12) \mathrm{mm}^{-1} ;[\mathrm{HbT}]=(24 \pm 10) \mu \mathrm{M}$; and $\mathrm{SatO}_{2}=(70 \pm 10) \%$. There was no significant difference in the optical properties or the [HbT] and $\mathrm{SatO}_{2}$ between the left and right breasts.

When each cohort was analysed separately, the only significant within-subject variation seen in the bulk $[\mathrm{HbT}]$ values in volunteers scanned once a week for a month $(p=0.022)$ (table 4). However, there is no clear pattern in the variation from week to week.

\subsection{Discussion}

There was no substantial statistical difference between images from the same woman taken daily, weekly or monthly. This demonstrates the basic reproducibility of the technique for longitudinal studies.

It has been shown by some groups that there is variation in the whole breast in response to systemic chemotherapy. Choe reported changes of over the course of treatment in a single subject, with a decrease in overall $\mathrm{SatO}_{2}$ from $(81.2 \pm 1.4) \%$ to $(59.9 \pm 0.6) \%$ to 
Table 4. The physiological parameters extracted from 3D images of the breast, assessing intrasubject variation in volunteers. The only significant variation seen was in the scan number for the $[\mathrm{HbT}]$ images for the women scanned once a week for a month. ${ }^{*}$ denotes $p \leqslant 0.05$.

\begin{tabular}{lll}
\hline & \multicolumn{2}{l}{$\begin{array}{l}p \text { value for difference } \\
\text { between scans }\end{array}$} \\
\cline { 2 - 3 } Imaging protocol & {$[\mathrm{HbT}](\mu \mathrm{M})$} & $\mathrm{SatO}_{2}(\%)$ \\
\hline Eight scans in one session & 0.146 & 0.593 \\
Weekly & $0.022^{*}$ & 0.438 \\
Monthly & 0.856 & 0.061 \\
\hline
\end{tabular}

$(61.1 \pm 1.0) \%$ during the last three cycles of chemotherapy and similar changes in overall [HbT] that varied with the patient's haematocrit (Choe et al 2005). Busch et al reported changes in the healthy tissue of three subjects using their probability of malignancy signature, based on physiological and optical properties (Busch et al 2013). These changes in the healthy breast tissue are not surprising as certain chemotherapy agents can cause anaemia in some patients, which will affect the whole body. Other studies have shown that the background tissue physiological properties remain relatively stable in some patients, while other patients show changes in the background breast physiological properties over the course of treatment (Enfield et al 2011).

There was a significant difference in images of $[\mathrm{HbT}]$ taken weekly $(p=0.022)$. This was entirely due to a single individual, which may explain a contradiction in literature where some studies show a change in optical properties with the menstrual cycle (Shah et al 2001, Pogue et al 2004) and some do not (Cubeddu et al 2000, Yates 2005a, Leff et al 2008). We intend to analyse this further by examining more women on a weekly basis over a longer period of time.

We saw no difference in the physiological properties of the left and right breast in seven out of the eight volunteers. This agrees with the published findings by Fang et al (2011) who found a strong correlation between the left and right breast $\left([\mathrm{HbT}](R=0.96)\right.$ and $\mathrm{SatO}_{2}(R=$ 0.89)) and Yates et al (2005c) who also noted right-left symmetry in the images from healthy pre-menopausal volunteers.

\section{Between subjects variation of optical images of the breast}

\subsection{Introduction}

There was significant variation in the raw data when comparing different subjects, so it can be assumed that there will be variation in the images as well. There is also extensive published data in the literature on the effects of BMI, breast size and radiographic density on NIR images and data, particularly [HbT] (Srinivasan et al 2003, Pogue et al 2004).

\subsection{Methods}

The same method that was used for the within-subject variation has also been used to look for significant differences between subjects.

\subsection{Results}

There were significant between-subject variations in all three scanning protocols for [HbT] (table 5). There is no significant variation in $\mathrm{SatO}_{2}$. 
Table 5. The physiological parameters extracted from 3D images of the breast, assessing intersubject variation in volunteers. There were significant differences between all the [HbT] images when comparing different volunteers. ${ }^{*} p \leqslant 0.05$.

\begin{tabular}{lll}
\hline & \multicolumn{2}{l}{$\begin{array}{l}p \text { value for differences } \\
\text { between volunteers }\end{array}$} \\
\cline { 2 - 3 } Imaging protocol & {$[\mathrm{HbT}](\mu \mathrm{M})$} & $\mathrm{SatO}_{2}(\%)$ \\
\hline Eight scans in one session & $0.024^{*}$ & 0.061 \\
Weekly & $0.016^{*}$ & 0.372 \\
Monthly & $0.026^{*}$ & 0.128 \\
\hline
\end{tabular}

\subsection{Discussion}

In the reconstructed images, there was significant variation in the [HbT] images for all three imaging protocols when comparing subjects, but not in the $\mathrm{SatO}_{2}$

A meta-analysis by Leff et al (2008) calculated global averages for breast tissue in healthy pre-menopausal women of $[\mathrm{HbT}]=(34 \pm 9) \mu \mathrm{M}$ and $\mathrm{SatO}_{2}=(75 \pm 2) \%$. These results were calculated from just two studies, who individually report [HbT] averages of [HbT] and $\mathrm{SatO}_{2}$ of $(40 \pm 3) \mu \mathrm{M}$ and $(73 \pm 6) \%$ and $27 \mu \mathrm{M}$ and $77 \%$ respectively (Cerussi et al 2001, 2002). Our results show an average of $[\mathrm{HbT}]$ and $\mathrm{SatO}_{2}((24 \pm 10) \mu \mathrm{M}$ and $(70 \pm 10) \%$ respectively. When these data are pooled and weighted by the number of women scanned the weighted average $[\mathrm{HbT}]$ and $\mathrm{SatO}_{2}$ are $(29 \pm 8) \mu \mathrm{M}$ and $(73 \pm 3) \%$ respectively.

Brooksby et al (2006) found that there was significant variation between the [HbT] of adipose and glandular breast tissue but there was no significant difference in $\mathrm{SatO}_{2}$ when examining 11 different subjects.

We note that the S.D. of $\mathrm{SatO}_{2}$ in published studies expressed as a percentage is $4 \%$ whereas that of $[\mathrm{HbT}]$ is much higher at $28 \%$. This is likely to be the reason why we saw significant changes in $[\mathrm{HbT}]$ but not $\mathrm{SatO}_{2}$. Also, only two wavelengths were used to obtain data and derived the physiological parameters. It is known that $\mathrm{SatO}_{2}$ measurements are mainly associated with the post-processing which makes the values less reliable than [HbT].

\section{Conclusions}

This study aimed to investigate the effect of sources of variation in data and images that may occur when using the UCL NIR breast imaging system and patient interface, using data obtained from eight healthy pre-menopausal volunteers. Both within- and between-subject variation were examined, as well as systematic variation.

There are obviously some limitations in this study and in the analysis. The number of volunteers studied is small. We do not have a measure of breast density for the volunteers that took part in this study, which would help interpret any differences in [HbT] between subjects.

In conclusion, this study shows that the greatest source on variation in optical mammography is due to differences between subjects. There is no significant variation between the left and right breast. The raw data and images from each individual subject are reproducible, even over a time period of several months. This means in studies of treatment response in breast cancer patients, we can be reassured that any changes in [HbT] or $\mathrm{SatO}_{2}$ within the tumour region may be due to a genuine response to treatment, as the background breast tissue physiological properties remains relatively constant. However, as discussed, changes have been seen in the physiological of the rest of the breast as well as the tumour in some patients being treated with neoadjuvant chemotherapy. 


\section{Acknowledgments}

The authors would like to thank the volunteers who took part in this study. This work was funded by Cancer Research UK and Engineering and Physical Sciences Research Council (EPSRC) as part of the KCL/UCL Comprehensive Cancer Imaging Centre.

\section{References}

Arridge S R and Lionheart W R B 1998 Nonuniqueness in diffusion-based optical tomography Opt. Lett. 23 882-4 Arridge S R and Schweiger M 1997 Image reconstruction in optical tomography Phil. Trans. R. Soc. B 352 717-26 Brooksby B, Pogue B W, Jiang S, Dehghani H, Srinivasan S, Kogel C, Tosteson T D, Weaver J, Poplack S P and Paulsen K D 2006 Imaging breast adipose and fibroglandular tissue molecular signatures by using hybrid MRI-guided near-infrared spectral tomography Proc. Natl Acad. Sci. USA 103 8828-33

Busch D et al 2013 Optical malignancy parameters for monitoring progression of breast cancer neoadjuvant chemotherapy Biomed. Opt. Express 4 105-21

Cerussi A E, Berger A J, Bevilacqua F, Shah N, Jakubowski D, Butler J, Holcombe R F and Tromberg B J 2001 Sources of absorption and scattering contrast for near-infrared optical mammography Acad. Radiol. 8 211-8

Cerussi A E, Jakubowski D, Shah N, Bevilacqua F, Lanning R, Berger A J, Hsiang D, Butler J, Holcombe R F and Tromberg B J 2002 Spectroscopy enhances the information content of optical mammography J. Biomed. Opt. 7 60-71

Choe R and Durduran T 2012 Diffuse optical monitoring of the neoadjuvant breast cancer therapy IEEE J. Sel. Top. Quantum Electron. 18 1367-86

Choe $\mathrm{R}$ et al 2005 Diffuse optical tomography of breast cancer during neoadjuvant chemotherapy: a case study with comparison to MRI Med. Phys. 32 1128-39

Cubeddu R, D'Andrea C, Pifferi A, Taroni P, Torricelli A and Valentini G 2000 Effects of the menstrual cycle on the red and near-infrared optical properties of the human breast Photochem. Photobiol. 72 383-91

Dehghani H, Brooksby B A, Pogue B W and Paulsen K D 2005 Effects of refractive index on near-infrared tomography of the breast Appl. Opt. 44 1870-8

Ding H, Lu J Q, Jacobs K M and Hu X-H 2005 Determination of refractive indices of porcine skin tissues and intralipid at eight wavelengths between 325 and $1557 \mathrm{~nm}$ J. Opt. Soc. Am. A 22 1151-7

Durduran T, Choe R, Culver J P, Zubkov L, Holboke M J, Giammarco J, Chance B and Yodh A G 2002 Bulk optical properties of the healthy female breast tissue Phys. Med. Biol. 47 2847-61

Enfield L C, Cantanhede G, Westbroek D, Douek M, Purushotham A D, Hebden J C and Gibson A P 2011 Monitoring the response to primary medical therapy for breast cancer using three-dimensional time-resolved optical mammography Technol. Cancer Res. Treat. $10533-47$

Enfield L C, Gibson A P, Everdell N L, Delpy D T, Schweiger M, Arridge S R, Richardson C, Keshtgar M, Douek M and Hebden J C 2007 Three-dimensional time-resolved optical mammography of the uncompressed breast Appl. Opt. 46 3628-38

Enfield L C, Gibson A P, Hebden J C and Douek M 2009 Optical tomography of breast cancer-monitoring response to primary medical therapy Targeted Oncol. 4 219-33

Fang Q, Carp S A, Selb J, Boverman G, Xhang Q, Kopans D B, Moore R H, Miller E L, Brooks D H and Boas D A 2009 Combined optical imaging and mammography of the healthy breast: optical contrast derived from breast structure and compression IEEE Trans. Med. Imaging 28 30-42

Fang Q, Selb J, Carp S A, Boverman G, Miller E L, Brooks D H, Moore R H, Kopans D B and Boas D A 2011 Combined optical and x-ray tomosynthesis breast imaging Radiology 258 89-97

Hebden J C and Delpy D T 1994 Enhanced time resolved imaging using a diffusion model of photon transport Opt. Lett. $19311-3$

Hebden J C, Gonzalez F M, Gibson A, Hillman E M C, Yusof R, Everdell N, Delpy D T, Zaccanti G and Martelli F 2003 Assessment of an in situ temporal calibration method for time-resolved optical tomography J. Biomed. Opt. 8 87-92

Hillman E M C 2002 Experimental and theoretical investigations of near-infrared tomographic imaging methods and clinical applications PhD Thesis University of London

Jakubowski D B, Cerussi A E, Bevilacqua F, Shah N, Hsiang D, Butler J and Tromberg B J 2004 Monitoring neoadjuvant chemotherapy in breast cancer using quantitative diffuse optical spectroscopy: a case study J. Biomed. Opt. $9230-8$

Jennions D K 2008 Time-resolved optical tomography instrumentation for fast 3D functional imaging PhD Thesis University of London 
Jiang S et al 2009 Evaluation of breast tumor response to neoadjuvant chemotherapy with tomographic diffuse optical spectroscopy: case studies of tumor region-of-interest changes Radiology 252 551-60

Leff D R, Warren O, Enfield L C, Gibson A P, Athanasiou T, Pattern D K, Hebden J C, Yang G Z and Darzi A 2008 Diffuse optical imaging of the healthy and diseased breast-a systematic review Breast Cancer Res. Treat. 1089

Matcher S J, Cope M and Delpy D T 1994 Use of the water absorption spectrum to quantify tissue chromophore concentration changes in near-infrared spectroscopy Phys. Med. Biol. 38 177-96

Pogue B W, Jiang S, Dehghani H, Kogel C, Soho S, Srinivasan S, Song X, Tosteson T D, Poplack S P and Paulsen K D 2004 Characterization of hemoglobin, water, and NIR scattering in breast tissue: analysis of intersubject variability and menstrual cycle changes J. Biomed. Opt. 9 541-52

Schmidt F E W, Fry M E, Hillman E M C, Hebden J C and Delpy D T 2000 A 32-channel time-resolved instrument for medical optical tomography Rev. Sci. Instrum. 71 256-65

Shah N, Cerussi A, Eker C, Espinoza J, Butler J, Fishkin J, Hornung R and Tromberg B 2001 Noninvasive functional optical spectroscopy of human breast tissue Proc. Natl Acad. Sci. USA 98 4420-5

Spinelli L, Torricelli A, Pifferi A, Taroni P, Danesini G M and Cubeddu R 2004 Bulk optical properties and tissue components in the female breast from multiwavelength time-resolved optical mammography J. Biomed. Opt. 9 1137-42

Srinivasan S, Pogue B W, Jiang S, Dehghani H, Kogel C, Soho S, Gibson J J, Tosteson T D, Poplack S P and Paulsen K D 2003 Interpreting hemoglobin and water concentration, oxygen saturation, and scattering measured in vivo by near-infrared tomography Proc. Natl. Acad. Sci. USA 100 12349-54

Yates T D 2005a Time-resolved optical tomography for the detection and specification of breast disease PhD Thesis University of London

Yates T D, Hebden J C, Gibson A P, Enfield L C, Everdell N L, Arridge S R and Delpy D T 2005b Time-resolved optical mammography using a liquid coupled interface J. Biomed. Opt 10054011

Yates T D, Hebden J C, Gibson A P, Everdell N L, Arridge S R and Douek M 2005c Optical tomography of the breast using a multi-channel time-resolved imager Phys. Med. Biol. 50 2503-17 